\title{
The cranium as an oscillator: analysis of phase relationships in intracranial blood and CSF pulsations using flow sensitive MRI Mark Wagshul*1, Michael Egnor ${ }^{2}$, Erin McCormack ${ }^{1}$, Pat McAllister ${ }^{3}$ and Raphael Hazel ${ }^{4}$
} \author{
6, Room 140, Stony Brook, New York 11794-8661, USA \\ Email: Mark Wagshul* - mark.wagshul@stonybrook.edu \\ * Corresponding author \\ from 50th Annual Meeting of the Society for Research into Hydrocephalus and Spina Bifida \\ Cambridge, UK. 30 August - 2 September 2006 \\ Published: 2I December 2006 \\ Cerebrospinal Fluid Research 2006, 3(SuppI I):SI2 doi:I0.II86/I743-8454-3-SI-SI 2
}

Address: ${ }^{1}$ Department of Radiology, State University of New York at Stony Brook, Health Science Center, Level 4, Room 109, Stony Brook, New York 11794-8460, USA, ${ }^{2}$ Department of Neurosurgery, State University of New York at Stony Brook, Health Science Center, T-12 Room 080, Stony Brook, New York 11794-8122, USA, ${ }^{3}$ Department of Neurosurgery, Wayne State University School of Medicine, 4201 St. Antoine, Suite UHC-6E, Detroit, Michigan 48201, USA and ${ }^{4}$ Department Physiology \& Biophysics, State University of New York at Stony Brook, Health Science Center, T-

(C) 2006 Wagshul et al; licensee BioMed Central Ltd.

\section{Background}

Over the past two decades, flow sensitive MRI has been used to demonstrate phase relationships between waveforms of blood and CSF pulsations in the cranium and marked changes in phase of CSF pulsatility have been described in hydrocephalus. However, there is no systematic explanation for the normal phase relationships between blood and CSF pulsations, and we have no theoretical framework for understanding these phase relationships in normal subjects and no theoretical framework for understanding why they change in hydrocephalus. We have undertaken a systematic study of the phase relationships of intracranial blood and CSF flow in normal individuals using flow sensitive MRI, and interpret the results using a model of the cranium as a forced oscillator.

\section{Materials and methods}

Fifteen healthy volunteers were studied on a 1.5T Philips Edge scanner. Arterial flow into the cranium was used as the reference waveform. Phase contrast images were collected using an encoding velocity of $5 \mathrm{~cm} / \mathrm{s}$ for the CSF images and $60 \mathrm{~cm} / \mathrm{s}$ for the vascular study. After Fourier transformation of the flow waveform from each CSF region, the phase was determined from the phase of the fundamental harmonic frequency of the flow waveform.

\section{Results}

A clear phase lag of velocity was demonstrated in the cerebral aqueduct $(-55.3 \pm 15.8$ degrees $)$ as well as in the prepontine cistern $(-23.9 \pm 7.6$ degrees $)$ relative to the phase in the carotids. In comparison, the flow phase at the craniocervical junction was zero $(4.4 \pm 10.8$ degrees $)$, i.e. a pulse waveform that is synchronous with the arterial input waveform. Sagittal images showed a progressively decreasing phase lag moving from the prepontine region down to the midbrain and spine levels.

\section{Conclusion}

Based on the oscillator model, a CSF phase lag occurs due to low elastance (high compliance) with respect to the inertia of the pulse, such as would occur in the ventricular system. A CSF phase lead would occur in a region with low inertia with respect to elastance, which would occur in a cyst and has been described as the "rebound sign". Zero phase at the foramen magnum represents high impedance resonance, and may play a role in the intracranial windkessel mechanism. In this theoretical model, changes in compliance in the subarachnoid spaces would alter pulsatile intracranial dynamics, particularly the windkessel mechanism, and would account for several important dynamic features characteristic of clinical and experimental hydrocephalus. 